\title{
Application of Sparse Non-Negative Matrix Factorization in infrared non- destructive testing
}

\author{
by Bardia Yousefi, Clemente Ibarra Castanedo, Xavier P.V. Maldague
}

Computer vision and systems laboratory (CVSL), Department of Electrical and Computer Engineering, Laval University, Quebec City (Quebec) G1V 0A6, Canada

Email:Bardia.Yousefi.1@ulaval.ca ,\{llbarraC,Xavier.Maldague\}@gel.ulaval.ca

\begin{abstract}
Non-negative matrix factorization (NMF) solves the problem of negative basis in principal component analysis (PCA) and widely used in diverse applications in different fields. Here, we show an application of sparse-NMF in infrared non-destructive testing (IR-NDT) imaging. We applied Sparse-NMF to determine the subsurface defects of an Aluminium plate specimen applying active thermographic method. To obtain results we compared the ability of Sparse-NMF to detect subsurface defects and its computational load in compared to state-of-the-art thermographic approaches such as: principal component analysis/thermography (PCT), Candid Covariance-Free Incremental Principal Component Thermography (CCIPCT), Sparse PCT, non-negative matrix factorization (NMF), and standard NMF with gradient descend (GD) and nonnegative least square (NNLS). The results show considerable performance (93\%- 39.52s) for Sparse-NMF, which conclusively indicate the promising performance as a confirmation for the outlined properties.

Keywords: Subsurface defect detection, Sparse Non-negative matrix factorization (Sparse-NMF), infrared non-destructive testing (IR-NDT).
\end{abstract}

\section{SUMMARY}

Non-negative matrix factorization (NMF) is a matrix decomposition approach similar to PCA with a difference in basis matrix which decomposes an input matrix into two low-rank non-negative matrices [1]. Nonnegative property of NMF is not followed by PCA (basis matrix produced by PCA can have negative parameters). NMF's origin is traced back to 1970s ([2-4]) which has been studied extensively. In 2001, Lee and Seung [1,2] presented NMF applying for clustering and afterward NMF became more known for data-mining and machine learning uses and showed its ability for solving challenging pattern recognition problems. Matrix factorization has been employed for infrared non-destructive testing (IRNDT) where showed the application of three methods: principal component analysis (PCA), non-negative matrix factorization (NMF), and archetypal analysis (AA) and showed its advantages and pitfalls [5]. Moreover, the application of NMF for two ways of its computations using gradient descend (GD) and non-negative least square (NNLS) for evaluating of cultural heritage objects and buildings illustrated considerable performance of such algorithm for detecting subsurface defects [6].

Here, we show the application of sparse non-negative matric factorization (Sparse-NMF) in detecting subsurface defects. Aluminium (AL) plate has been previously utilized for the evaluation of thermography techniques and represented a substantial tool of benchmarking thermographic methods. The data-set was compiled using following experimental scenario regarding acquisition parameters. The inspection was conducted from the front side of the specimen (having the depths range from 0.2 to $1 \mathrm{~mm}$ ). Two photographic flashes were used: Balcar FX 60, 5ms thermal pulse, $6.4 \mathrm{~kJ} / \mathrm{flash}$. Infrared camera: Santa Barbara Focal plane (MWIR, nitrogen cooled, InSb, $320 \times 256$ pixels). The parameters of acquisition are tuned as: Sampling rate, $\boldsymbol{f}_{\boldsymbol{s}}=157 \mathrm{~Hz}$; Duration, $\boldsymbol{t}_{\boldsymbol{a c q}}=6.37 \mathrm{~s}$; Time step, $\boldsymbol{D}_{\boldsymbol{t}}=0.025 \mathrm{~s}$; Truncation window, $w(t)_{s}=6.37 \mathrm{~s}$; Total number of frames $=250$. Figure 1.h illustrates the scheme of the specimen which has been utilized [7]. The presented thermography analysis has been performed in a PC (Intel(R) Core(TM) i5 CPU, 3.20GHz, RAM 16.00GB, 64 bit Operating System) and using MATLAB computer programming. Figure 1 presents selected results of the subsurface defect detection using Sparse-NMF (Figure 1.f) compared with state-of-the-art approaches such as PCT [8] (Figure 1.b), Candid Covariance-Free Incremental Principal Component Thermography (CCIPCT) [9] (Figure 1.g), NMF (Figure 1.a), NMF-gd (Figure 1.c), NMF-nnls [6] (Figure 1.d), and Sparse-PCT [10] (Figure 1.e). The qualitative results of Sparse-NMF indicated considerable accuracy relative to state-of-the-art techniques. To calculate the quantitative accuracy, we employed a binary image as ground truth (GT) as a reference for our calculation. A metric called Intersection over Union (loU), also denoted as Jaccard index, is used to quantify the percent overlap between the GT and our prediction output and measured by the number of pixels common between the GT and resulting masks divided by the overall number of pixels existing across both masks. Figure 1.i presents quantitative assessment of Sparse-NMF in comparison to stateof-the-art thermographic approaches and showed $93 \%$ loU accuracy with 39.52 s computational load. The NMF and PCT have a loU range of $95 \%(0.25 \mathrm{~s})$ and $91 \%(0.56 \mathrm{~s})$, respectively. NMF-gd and NMF-nnls were likewise relatively accurat having $87 \%(14.81 s)$ and $83 \%(45.18 s)$, respectively. 


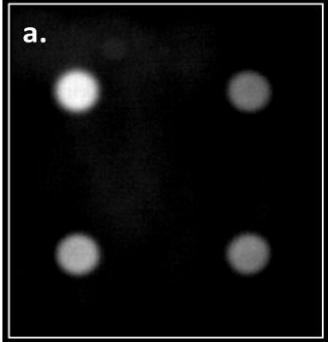

NMF

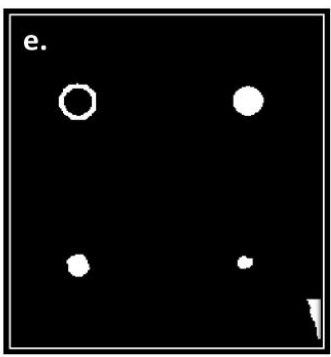

Sparse-PCT

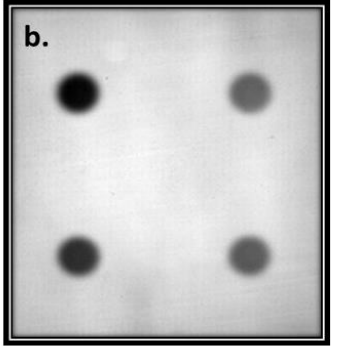

PCT

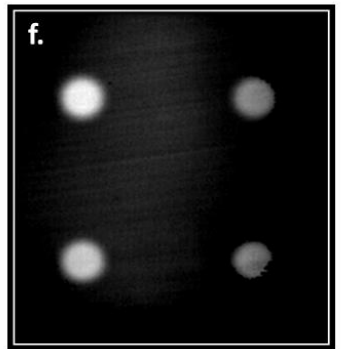

Sparse-NMF

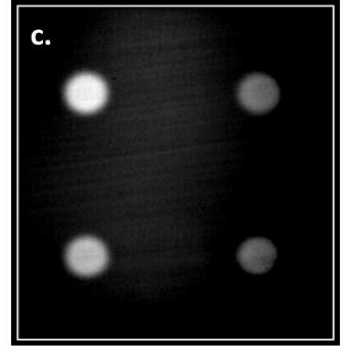

NMF-gd

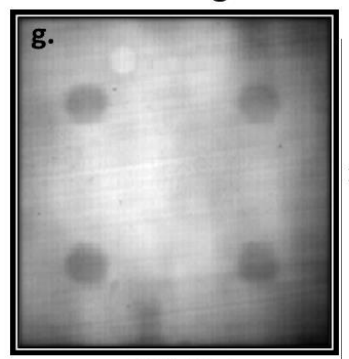

CCIPCT

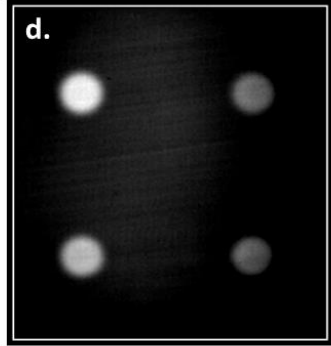

NMF-nnls

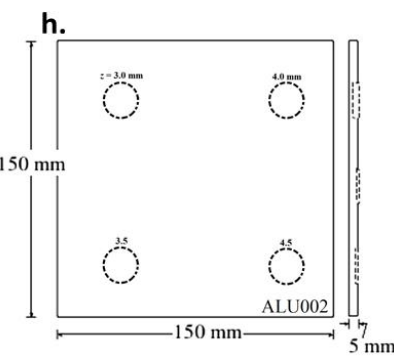

i.

Accuracy and computational time of approaches for Aluminium specimen

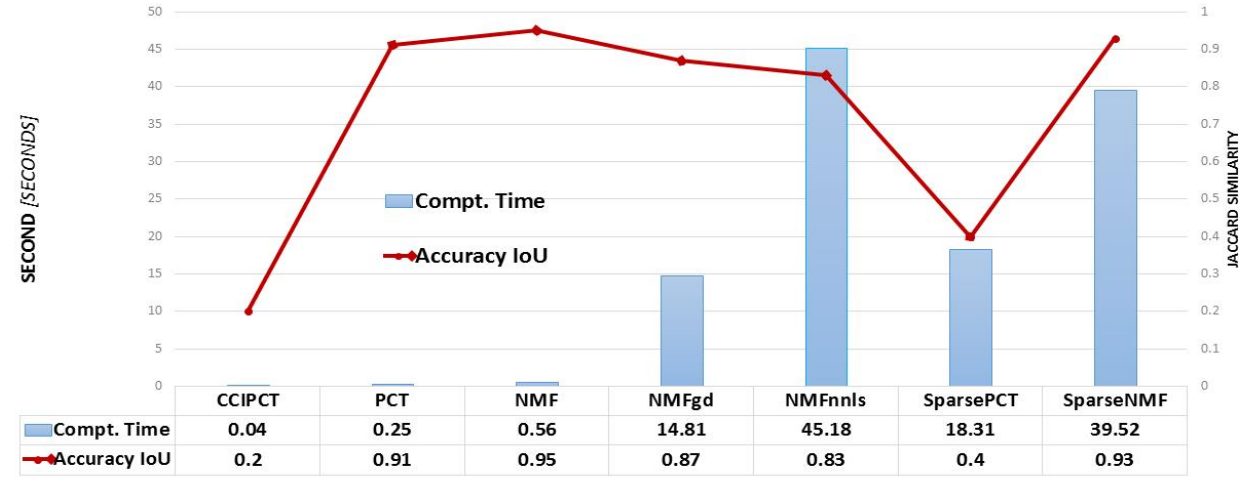

Fig.1 Evaluation of the qualitative accuracy of Sparse-NMF(f.) versus state-of-the-art approaches(a-e,g) in thermography. The graph (i.) shows the loU metric and computational load of each algorithm.

The quantitative results of Sparse-PCT and CCIPCT for detecting defects were not high (unlike their qualitative results), probably due to smaller targeted defects area and lower resolution of detected surface. To conclude, Sparse-NMF showed significant accuracy for detecting the subsurface defects and can be considered as an extensive technique for thermographic analysis.

\section{REFERENCES}

[1] D. D. Lee and H. S. Seung, "Learning the parts of objects by nonnegative matrix factorization," Nature, vol. 401, no. 6755, p. $788,1999$.

[2] A. Berman and R. J. Plemmons, "Inverses of nonnegative matrices," Linear and Multilinear Algebra, vol. 2, no. 2, pp. 161-172, 1974.

[3] D. D. Lee and H. S. Seung, "Algorithms for non-negative matrix factorization," in Advances in neural information processing systems, 2001, pp. 556-562.

[4] P. Paatero and U. Tapper, "Positive matrix factorization: A non-negative factor model with optimal utilization of error estimates of data values," Environmetrics, vol. 5, no. 2, pp. 111-126, 1994.

[5] Marinetti, S., Finesso, L., \& Marsilio, E. (2006). Matrix factorization methods: Application to thermal NDT/E. NDT \& E International, 39(8), 611-616.

[6] Yousefi, B., Sfarra, S., Ibarra-Castanedo, C., Avdelidis, N. P., \& Maldague, X. P. (2019). Thermography data fusion and nonnegative matrix factorization for the evaluation of cultural heritage objects and buildings. Journal of Thermal Analysis and Calorimetry, 136(2), 943-955.

[7] Ibarra-Castanedo, C., \& Maldague, X. P. (2004, April). Defect depth retrieval from pulsed phase thermographic data on plexiglas and aluminum samples. In Thermosense XXVI (Vol. 5405, pp. 348-357). International Society for Optics and Photonics.

[8] N. Rajic, Principal component thermography, Tech. rep., DTIC Document (2002).

[9] Yousefi, B., Sfarra, S., Castanedo, C. I., \& Maldague, X. P. (2017). Comparative analysis on thermal non-destructive testing imagery applying Candid Covariance-Free Incremental Principal Component Thermography (CCIPCT). Infrared Physics \& Technology, 85, 163169.

[10] Yousefi, B., Sfarra, S., Sarasini, F., Castanedo, C. I., \& Maldague, X. P. (2019). Low-Rank Sparse Principal Component Thermography (Sparse-PCT): Comparative assessment on detection of subsurface defects. Infrared Physics \& Technology. 